\title{
Things that go bump in the night: the curious case of NGC 660
}

\author{
Megan K. Argo* \\ Jodrell Bank Centre of Astrophysics, University of Manchester, M13 9PL, UK \\ E-mail: megan.argo@gmail.com
}

Ilse M. van Bemmel

JIVE, Postbus 2, 7990 AA Dwingeloo, The Netherlands

E-mail: bemmel@jive.nl

\section{Sam D. Connolly}

University of Southampton, Highfield, Southampton, SO17 1BJ, UK

E-mail: sdclg08@soton.ac.uk

\section{Robert J. Beswick}

Jodrell Bank Centre of Astrophysics, University of Manchester, M13 9PL, UK

E-mail: robert.beswick@manchester.ac.uk

\begin{abstract}
We report new EVN, e-MERLIN and WSRT observations of a recent outburst in the polar ring galaxy NGC 660. First reported in 2013, a significant increase in brightness has occurred within the galaxy, along with significant spectral changes. Archival VLA, MERLIN and VLBA observations show a ring of starburst-related emission in the main disk of the galaxy, but no evidence for a compact radio core prior to 2010. Our new observations show significant increase in brightness at low resolution (WSRT), and a new compact object located near the dynamical centre of the galaxy (e-MERLIN). High resolution imaging with the EVN shows this object consists of multiple spatial components. Spectra have been obtained at $1.4 \mathrm{GHz}$ with all three arrays, showing a consistent picture of multiple narrow HI absorption components seen against the new bright, compact core. The evidence points to a new period of AGN activity in this galaxy.
\end{abstract}

12th European VLBI Network Symposium and Users Meeting

7-10 October 2014

Cagliari, Italy

${ }^{*}$ Speaker. 


\section{A new radio source in NGC 660}

NGC 660 is a polar ring galaxy with a LINER-type nucleus (low-ionisation nuclear emissionline region), located $\sim 13 \mathrm{Mpc}$ away in Pisces. With a stellar population in the ring estimated to have an age of a few billion years [1], the galaxy has been well-studied by many authors, but has shown no sign of an active nucleus, and radio emission from previously-known compact sources in the disk appears to be purely starburst-related.

At radio wavelengths, NGC 660 was seen to flare spectacularly sometime between 2008 and 2012 and has been reported to have developed a new continuum source with a GHz-peak spectrum and a peak flux density of $\sim 0.5 \mathrm{Jy}$ at $5 \mathrm{GHz}$ [2]. High-resolution imaging has reportedly shown this increase to be due to a new source in the core, the nature of which is currently unclear. Simultaneously, $\mathrm{OH}$ emission and absorption in both the ground state $(18-\mathrm{cm})$ and excited $(6-\mathrm{cm})$ lines has developed, and $\mathrm{H}_{2} \mathrm{CO}$ absorption is seen against the new continuum source [2].

Archive VLA (Very Large Array) data at $8.4 \mathrm{GHz}$ from 1986 [3] shows a ring of starburstrelated emission in the main disk of the galaxy, but no compact emission at the position of the new radio source. Archival data we have obtained from other telescopes also show no evidence for a central radio object in this galaxy prior to 2010. Recently we have obtained new 1.4-GHz observations with the WSRT (Westerbork Synthesis Radio Telescope), e-MERLIN (electronic MultiElement Remote Linked Interferometer Network) and the EVN (European VLBI Network), and examined archival radio and Chandra data in order to investigate the nature of this source. Here we describe our current results and draw some conclusions on the likely cause of the event.

\section{New observations}

We obtained service-mode spectral line observations with the WSRT at $1.4 \mathrm{GHz}$ during 2013. These provide a direct comparison with archival WSRT data from 2009, and obtaining more than one observation allowed us to look for temporal changes on short time-scales. e-MERLIN observations were carried out at both 1.4 and $5 \mathrm{GHz}$ in May and June 2013. The observations used all six telescopes of the standard array, with 3C286 and OQ208 as the flux and point calibrators respectively, and J0143+1215 as the phase calibrator. Although the observations were carried out in continuum mode, the wide bandwidth of the new system $(512 \mathrm{MHz})$ enabled us to investigate the radio spectral energy distribution of the new object. More recent observations have been carried out utilising the spectral line capabilities of the new correlator, but these observations are not discussed here. A 12-hour EVN observation was carried out on October 30th 2013 using 11 telescopes of the array. 3C84 and 3C120 were used as bandpass calibrators, and J0143+1215 was again used as the phase calibrator. The data were correlated in two passes, one with eight sub-bands of 32 channels each for continuum, the other with one sub-band of 1024 channels for spectral line analysis.

\section{Archival data}

In addition to the new radio data obtained during 2013, we have also examined archival data from several telescopes. MERLIN observations from 1998 at both 1.4 and $5 \mathrm{GHz}$ have been reprocessed, and two VLBA (Very Long Baseline Array) observations from 2001 (5 GHz) and 2010 
$(8.4 \mathrm{GHz})$ have also been examined. The 2001 VLBA observation consisted of a total of three hours of data split over two hour angle ranges, whilst the 2010 data is two snapshot observations of eight minutes each, separated by 96 days. We have also re-processed the 1986 VLA A-array 8.4-GHz data from [3]. The Chandra archive also contains observations of NGC660. We have examined four epochs of data from January 2001, February 2003, and November \& December 2012. Unfortunately the pre-outburst data totals only $\sim 7 \mathrm{ks}$, insufficient to make a meaningful image. The post-outburst data totals $\sim 45 \mathrm{ks}$, allowing us to make an image and extract a spectrum.

\section{Observational results: a time-line}

The initial report of the flare in NGC 660 states that the event began some time between observations made with Arecibo in 2008 and 2012. Much of the available archive data was obtained before 2008, and all of the new observations were taken after the event was first reported in early 2013. Some of the archive data was taken between these two dates, however, so may provide a better constraint on when the event began.

The 1986 VLA data show radio emission from the starburst ring structure within the main disk of the galaxy. There is one prominent source, a known supernova remnant, visible in the north-eastern end of the ring, located some 2.5 " from the centre of the galaxy where the new object is located. While there are a handful of other weak sources, as well as some low-level extended emission, within the ring structure, there is no convincing evidence for a source at the position of the new radio source.

In 1998 NGC 660 was observed at both L- and C-band with six telescopes of MERLIN. The 1.4-GHz dataset shows diffuse emission from the starburst ring, with a velocity gradient across the disk showing textbook disk rotation; no strong radio source is apparent at the centre of the ring. Only one object is visible in the field at $5 \mathrm{GHz}$, the supernova remnant mentioned above. No flux is detected at the position of the new source to a $3-\sigma$ limit of $0.3 \mathrm{mJy} / \mathrm{beam}$.

The 2001 VLBA data shows no emission at $5 \mathrm{GHz}$ to a $3-\sigma$ limit of $0.41 \mathrm{mJy} / \mathrm{beam}$. The field of view of the observation is too small to contain the supernova remnant seen in the VLA data.

The 2001 and 2003 Chandra data are also not very helpful, consisting of only $\sim 7 \mathrm{ks}$ combined. The very low count rate $\left(1.30 \pm 0.10 \times 10^{-2} s^{-1}\right)$ meant that the exposure time was insufficient for useful analysis.

Archive WSRT observations from December 2009 have been examined [4]. These observations were made at $1.4 \mathrm{GHz}$ and include spectroscopy of the HI line. The resolution of the WSRT is insufficient to resolve the galaxy, but a point source is detected with a strong HI absorption signal. Because the galaxy is unresolved, we cannot be certain whether the outburst was already in progress by the time of this observation. These observations will be discussed in more detail in [4].

Despite consisting of single eight-minute scans, both of the VLBA observations in 2010 detect a compact $(1 \times 2$ mas) object at the centre of the galaxy. The $u-v$ coverage is insufficient to detect any spatial structure within the source, but a new compact object is clearly present in the data on both occasions. In the first epoch on September 16th 2010, two years before the first reported detection of a brightening of the galaxy in the radio, the object had a peak flux density of $18.2 \mathrm{mJy} / \mathrm{beam}$. The second epoch, on December $21 \mathrm{st}$, showed the flux density to have increased to $36.0 \mathrm{mJy} / \mathrm{beam}$. 


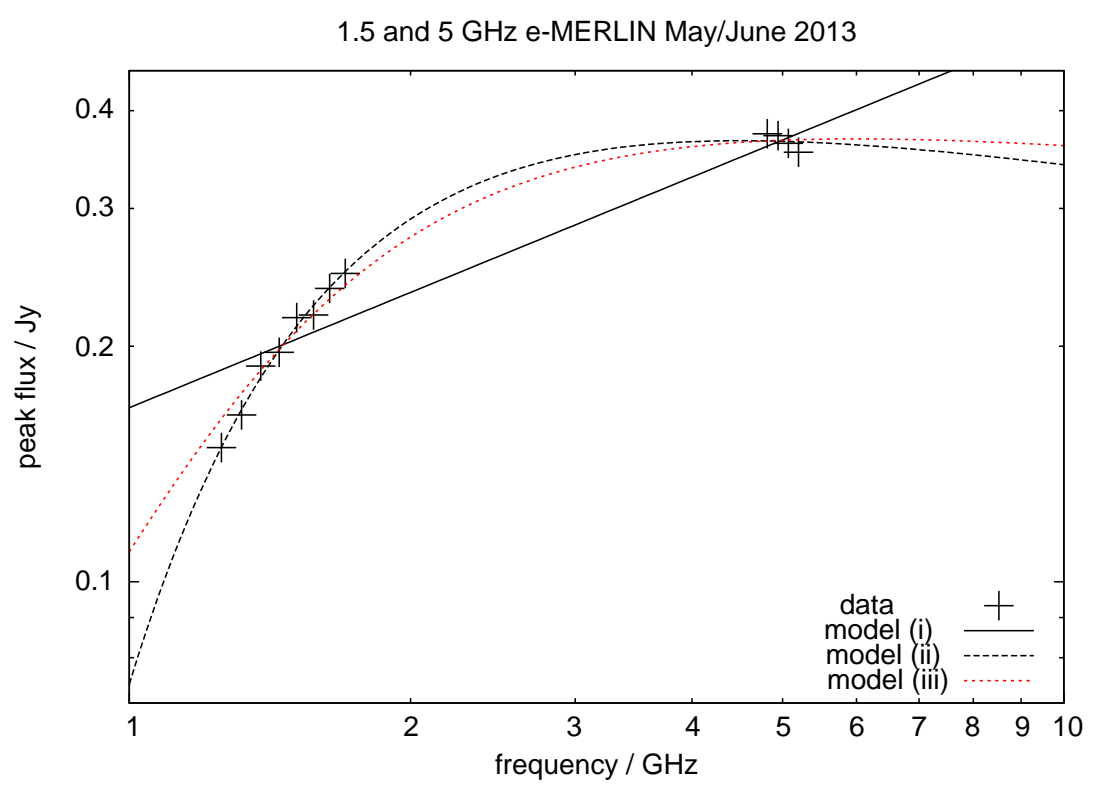

Figure 1: Peak flux measurements per sub-band from e-MERLIN observations made at 1.5 and $5 \mathrm{GHz}$ in May/June $2013(+)$. The lines shows least squares fits to the e-MERLIN data, with solid black, dashed black and dotted grey lines corresponding to a power law (i), a power law with free-free absorption by a foreground screen (ii), and a self-absorbed Bremsstrahlung model (iii).

The 2012 Chandra observations consist of $\sim 52 \mathrm{ks}$ of observing time. While the low count rate of the earlier data do not allow for a comparison of pre- and post-outburst data, the observations are still useful. There is extended diffuse X-ray emission aligned with the starburst disk of the galaxy, spatially matching well with the L-band emission seen in the 1998 MERLIN data, although the X-ray emission is more extended. A peak is seen at the position of the core, some 2-3 times brighter than the surrounding X-ray emission, but it is unclear whether this peak is due to the new radio source since a comparison with earlier data is not possible.

Our new WSRT observations in 2013 show an increase in the flux density of the galaxy compared to the 2009 WSRT observations. They also show a significant difference in the depth of the HI absorption signal, with the peak optical depth of the main velocity component being more than a factor of two greater than that measured in 2009 , and the appearance of a new velocity component at $900 \mathrm{~km} / \mathrm{s}[4]$.

The e-MERLIN observations in May and June 2013 show a strong central continuum source. Plotting the measured flux density in each sub-band ( 8 at L-band, 4 at C-band) shows the new radio source to have a Gigahertz-peaked spectrum (GPS) with a peak $\sim 3 \mathrm{GHz}$ (Fig. 1).

The EVN data taken in October 2013 show a bright compact source at the same coordinates as the VLBA detections in 2010. While the VLBA data did not resolve the source, our new observations clearly show that the source now has structure on mas scales [5] (Fig. 2).

\section{So what happened?}

Our new radio observations have confirmed the presence of a new radio source in the centre of the polar ring galaxy NGC 660, showing it to be a GPS source on e-MERLIN scales (Fig. 1), 

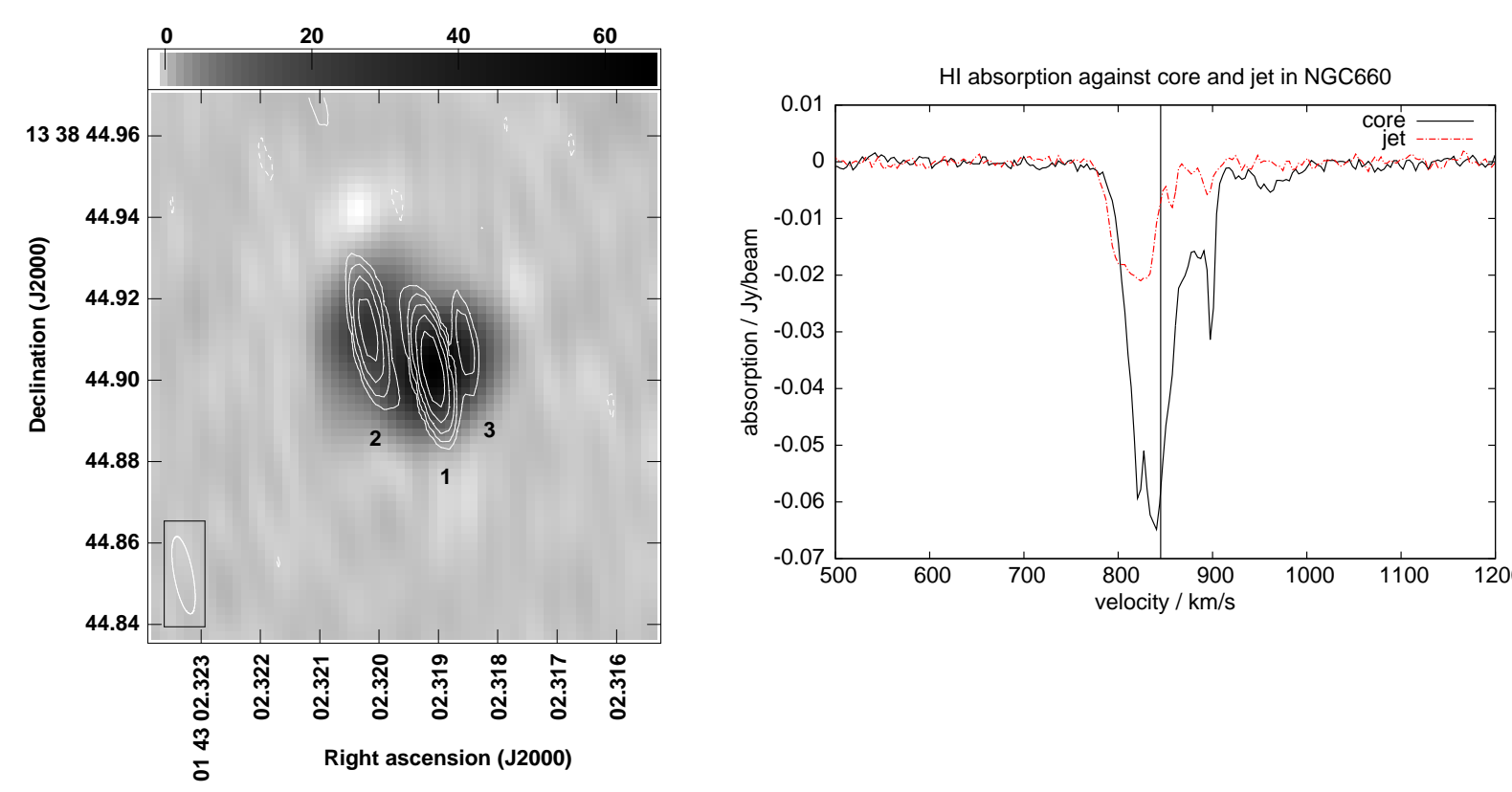

Figure 2: EVN results. Left: Continuum image of the new core emission in NGC 660 showing the jet-like components. "Low" resolution (natural weighting; $26 \times 20$ mas) image of the new components in the core of NGC 660 (grey scale), with contours from the "high" resolution (uniform weighting; $19 \times 6$ mas) image overlaid. The data are contoured at $(-1,1,2,4,8,16,32,64) \times 1.736 \mathrm{mJy} / \mathrm{beam}$, the greyscale range is -1.26 to $66.49 \mathrm{mJy} / \mathrm{beam}$, and the peak flux in the map is $55.5 \mathrm{mJy} / \mathrm{beam}$. Right: Spectrum against the core (black, solid) and jet (grey, dashed) components of NGC 660. The vertical line marks $845 \mathrm{~km} \mathrm{~s}^{-1}$ the systemic velocity of the galaxy.

with multiple velocity components in HI absorption, and an apparent core-jet morphology on VLBI scales (Fig. 2). At Galactic coordinates $(141,-47)$, the likelihood of a foreground Galactic flaring event is low, and a flare of this magnitude so far from the Galactic plane would be unlikely to have escaped attention. The galaxy could be undergoing a period of enhanced star-formation activity related to the encounter which caused the ring structure and starburst, although the $1.4 \mathrm{GHz}$ power detected in the EVN observations is significantly higher than any known individual radio supernova or remnant. The radio results would require some 10 to 100 "normal" radio supernovae within a region of $\sim 1.1 \mathrm{pc}$ in the space of a few years; a comparison with the well-known starburst Arp220, where the supernova rate is $4 \pm 2$ per year across a region almost $400 \mathrm{pc}$ in size [6], suggests that this explanation is also highly unlikely. A transient tidal disruption event is a possible explanation, although the lack of X-ray data (which should show a huge peak X-ray luminosity, variability by a factor of $\sim 200$, short rise time and long decay time, and an ultra-soft X-ray spectrum) makes this explanation difficult to accept or rule out. The characteristics of the radio source point to a new period of AGN activity as the most likely cause, turning NGC 660 from a LINER to a low luminosity AGN. Jets from LLAGN are more long-lived than those from tidal disruption events, so time will shed more light on the case. GPS sources represent an early stage in the evolution of radio galaxies, with the expanding lobes still contained within the host galaxy. 


\section{Conclusions}

We have confirmed the presence of a new radio source in the centre of the polar ring galaxy NGC 660. Initial reports suggested that the outburst began between 2008 and 2012, but analysis of archival data narrows this to between 2008.0 and 2010.7. The e-MERLIN observations at Land C-band show the source has a GPS radio spectrum with a turnover at $\sim 3 \mathrm{GHz}$. High angular resolution observations with the EVN show the source to be resolved, with what appears to be a bright core, along with a jet and possible counter-jet. If this is, as it appears to be, a new period of activity in the nucleus, then the current apparent size of the structure, together with the limits on the date of the beginning of this period of activity, can give us an estimate of the expansion speed. Assuming a distance to NGC 660 of $13 \mathrm{Mpc}$ [7], then the apparent speed of the jet is $0.6 \leq v \leq 1.2 c$. Further observations are planned in order to: measure the expansion speed, and hence place a better constraint on when the activity began; monitor how the radio SED evolves as the outburst progresses; further investigate the spectral line information which can be obtained with such a strong, narrow line of sight through the disk of the galaxy.

\section{Acknowledgements}

The European VLBI Network is a joint facility of European, Chinese, South African and other radio astronomy institutes funded by their national research councils. We thank the staff of JIVE, especially Bob Cambell, Zsolt Paragi, and Gabriele Surcis, for their assistance with the observations and correlation. We also thank Ian McHardy for useful discussions. The research leading to these results has received funding from the European Commission Seventh Framework Programme (FP/2007-2013) under grant agreement No. 283393 (RadioNet3). e-MERLIN is a National Facility operated by the University of Manchester at Jodrell Bank Observatory on behalf of STFC. This research has made use of v0.7 of the e-MERLIN pipeline, available at http://dx.doi.org/10.5281/zenodo.10163 The scientific results reported in this article are based in part on data obtained from the Chandra Data Archive. SDC thanks the STFC for support under a studentship.

\section{References}

[1] van Driel, W., Combes, F., Casoli, F. et al, 1995, Polar ring spiral galaxy NGC 660, AJ, 109, 942

[2] Minchin, R. F., Ghosh, T., Momjian, E. and Salter, C. J., 2013, A Radio Continuum and Spectral Line Outburst in the Nearby Galaxy NGC 660, American Astronomical Society Meeting Abstracts, 221, 157.06

[3] Carral, P., Turner, J. L. and Ho, P. T. P., 199015 GHz compact structure in galactic nuclei, ApJ, 362, 434

[4] van Bemmel et al in prep

[5] Argo, M. K., van Bemmel, I. M., Connolly, S. D. \& Beswick, R. J., 2015, MNRAS, in review

[6] Lonsdale, C. J., Diamond, P. J., Thrall, H., Smith, H. E. \& Lonsdale, C. J., 2006, ApJ, 647, 185

[7] Springbob, C. M., Masters, K. L., Haynes, M. P., Giovanelli, R. \& Marinoni, C., 2009, ApJ Supp. 182,474 